\title{
ON THE EXISTENCE OF ANALYTIC MAPPINGS
}

\author{
By Mitsuru Ozawa and Nobuyuki Suita
}

1. Let $R$ be an ultrahyperelliptic surface defined by $y^{2}=G(x)$ where $G(x)$ is an entire function having only an infinite number of simple zeros. Let $S$ be a similar surface defined by $y^{2}=g(x)$ with a similar $g(x)$ as $G(x)$. We already discussed the existence problem of non-trivial analytic mappings of $R$ into $S$ [4]. The following theorem is central theorem in the existence problem of non-trival analytic mappings.

THEOREM A. Suppose that there is a non-trivial analytic mapping of $R$ into $S$. Then there are entire $h(z)$ and meromorphic $f(z)$ satisfying $g \circ h(z)=f^{2}(z) G(z)$ and vice versa.

Hiromi and Mutō [2] proved the following

THEOREM B. Suppose that the order of $N(r, 0, G)$ is finite and the one of $N(r, 0, G)$ is finite positive and that there is a non-trivial analytic mapping of $R$ into $S$. Then the corresponding $h(z)$ is a polynomial of degree ord $N(r, 0, G)$ lord $N(r, 0, g)$.

In this paper we shall prove the following

THEOREM 1. Suppose that the assumptions of Theorem B are satisfied and further that $G$ is an entire periodic function of finite order. Then the existence of a non-trivial analytic mapping of $R$ into $S$ gives the following relation

$$
\operatorname{ord} G=\nu \text { ord } N(r, 0, g) \quad \nu= \begin{cases}1,2 & \text { if } \operatorname{ord} G \neq 2, \\ 1,2,3,4,6 & \text { if } \operatorname{ord} G=2 .\end{cases}
$$

There are examples which show the occurence of all the possible cases. In order to prove the above theorem we need several lemmas on number theory.

We shall give an application of Theorem 1 .

THEOREM 2. Besides the assumptions of Theorem 1 assume that $g$ is also periodic. Then every non-trivial analytic mapping of $R$ into $S$ reduces to a conformal mapping of $R$ onto $S$.

Our results do not depend on any representations of $R$ and $S$. We can for-

Recelved November 15, 1972. 
mulate them in an intrinsic manner. There are lots of related results. If $G(x)$ is $\left(e^{H(x)}-\gamma\right)\left(e^{H(x)}-\delta\right)$ with a polynomial $H(x)$ and $\gamma \delta(\gamma-\delta) \neq 0$, then $0<$ ord $g<\infty$ and the existence of non-trivial analytic mappings of $R$ into $S$ imply ord $G=\nu \operatorname{ord} g$, $\nu=1$ or 2 [5]. Rényi [6] proved that $g \circ h(z)$ with periodic $G(z)$ and a polynomial $h(z)$ implies $\operatorname{deg} h=1$ or 2 . In this tendency we can extend the Rényi's result.

THEOREM 3. Let $G(z)$ be an entire periodic function, $g(z)$ a non-constant entire function and $p(z), Q(z)$ polynomials. If $g \circ P(z)=Q(z) G(z)$, then $\operatorname{deg} P \leqq 2$.

THEORE 4. Let $G(z)$ be an entire periodic function of finite order and with only simple zeros. Let $g(z)$ be an entire function of non-zero finite order and with only simple zeros. Let $f(z)$ be an entire function of order less than $G$. If $g \circ P(z)$ $=f(z)^{2} G(z)$ with entire $P(z)$, then $P(z)$ is a polynomial of degree less than 3 .

\section{Lemmas on number theory}

LEMMA 1. The cyclotomic equation is irreducible over the rational number field.

LEMMA 2. The degree of the cyclotomic equation, which corresponds to the primitive $n^{\text {th }}$ roots of unity, is larger than 2 , unless $n=1,2,3,4,6$.

Lemma 2 is very easy to prove by using the so-called Euler's function $\phi(n)$. See [3], [7] for Lemmas 1 and 2.

Lemma 3. Let $\eta$ be $\exp (2 \pi i / n)$. If $n \neq 1,2,3,4,6$, then there are infinitely many triples of integers $p, q, r$ such that

for any given $\varepsilon>0$.

$$
0<\left|p+q \eta+r \eta^{2}\right|<\varepsilon
$$

Proof of Lemma 3. Assume that

Then

$$
p+q \eta+r \eta^{2}=0 .
$$

$$
\cos \frac{2 \pi}{n}=-\frac{q}{2 r}, \quad p=r
$$

Since $\eta$ and $\bar{\eta}$ are $n^{\text {th }}$ primitive roots of unity,

$$
\begin{aligned}
x^{2}-(\eta+\bar{\eta}) x+\eta \bar{\eta} & =x^{2}-2 x \cos \frac{2 \pi}{n}+1 \\
& =x^{2}+\frac{q}{r} x+1
\end{aligned}
$$

should be a factor of the cyclotomic equation. By Lemmas 1 and $2 n$ should be $1,2,3,4,6$ in this case. Hence for $n \neq 1,2,3,4,6$

$$
p+q \eta+r \eta^{2} \neq 0
$$


for any non-zero triple $(p, q, r)$. Since $\cos (2 \pi / n)$ is irrational for $n \neq 1,2,3,4,6$, we have

$$
0<\left|q \sin \frac{2 \pi}{n}+2 r \cos \frac{2 \pi}{n} \sin \frac{2 \pi}{n}\right|<\frac{\varepsilon}{2}
$$

for an infinite number of pairs $(q, r)$. Take $p=r$. Then

$$
0<\left|p+q \cos \frac{2 \pi}{n}+r \cos \frac{4 \pi}{n}\right|<\frac{\varepsilon}{2} \text {. }
$$

Thus for an infinite number of triples $(p, q, r), p=r$

$$
0<\left|p+q \eta+r \eta^{2}\right|<\varepsilon
$$

3. Proof of Theorem 1. We may start from

$$
g \circ h(z)=f^{2}(z) G(z) .
$$

Here we may take $g$ as the canonical product of its zeros. By Hiromi-Muto's theorem $\mathrm{B} h(z)$ is a polynomial of degree $n$ such that

$$
\text { ord } N(r, 0, G)=n \text { ord } N(r, 0, g) \text {. }
$$

Further since $G$ and $g$ have only simple zeros $f(z)$ is entire and has a finite number of zeros. We may assume that

$$
h(z)=z^{n}+a_{p} z^{p}+\cdots+a_{0}, \quad a_{p} \neq 0,1 \leqq p \leqq n-2 .
$$

Let $\tau$ be the period of $G$. The assumption $a_{p} \neq 0$ leads us to a contradiction. This is our first aim. Let $z_{\jmath}$ be the $j^{\text {th }}$ roots of $h(z)=w$. If $w$ is sufficiently large, then $z_{\jmath} \neq z_{k}$ for $j \neq k$. Further

$$
z_{\jmath}=\eta^{\jmath-1} w^{1 / n}-\frac{a_{p}}{n} \frac{1}{\eta^{-(p+1)(\jmath-1)} w^{(n-p-1) / n}}-\frac{a_{p-1}}{n} \frac{1}{\eta^{-p(\jmath-1)} w^{(n-p) / n}}+O\left(\frac{1}{w^{(n-p+1) / n}}\right) .
$$

Let $\phi\left(z_{1}\right)$ be $z_{2}$ and $\phi^{-1}\left(z_{2}\right)=z_{1}$. Consider

$$
\phi^{-1}\left(\phi\left(\phi^{-1}\left(\phi\left(z_{1}+\tau\right)+\tau\right)-\tau\right)-\tau\right) \equiv L\left(z_{1}\right) .
$$

Then with $\eta=\exp (2 \pi i / n)$

$$
\begin{aligned}
z_{2} & =\phi\left(z_{1}\right) \\
& =\eta z_{1}+\frac{a_{p}}{n} \frac{\eta-r_{i}^{p+1}}{z_{1}^{n-p-1}}+\frac{a_{p-1}}{n} \frac{\eta-\eta^{p}}{z_{1}^{n-p}}+O\left(\frac{1}{z_{1}^{n-p+1}}\right), \\
z_{1} & =\phi^{-1}\left(z_{2}\right) \\
& =\frac{z_{2}}{\eta}-\frac{a_{p}}{n} \frac{\eta-\eta^{p+1}}{\eta^{p+2}} \frac{1}{z_{2}^{n-p-1}}-\frac{a_{p-1}}{n} \frac{\eta-\eta^{p}}{\eta^{p+1}} \frac{1}{z_{2}^{n-p}}+O\left(\frac{1}{z_{2}^{n-p+1}}\right)
\end{aligned}
$$

and 


$$
\begin{aligned}
L\left(z_{1}\right)=z_{1} & +\frac{a_{p}}{n} \frac{\eta-\eta^{p+1}}{\eta} \tau \frac{\sum_{\substack{n=0 \\
j=0}}^{n-2}\left(z_{1}+\tau+\tau / \eta\right)^{\jmath}\left(z_{1}+\gamma\right)^{n-p-2-\jmath}}{\left(z_{1}+\tau\right)^{n-p-1}\left(z_{1}+\tau+\tau / \eta\right)^{n-p-1}} \\
& \quad-\frac{a_{p}}{n} \frac{\eta-\eta^{p+1}}{\eta} \tau \eta^{n-1} \frac{\sum_{\substack{n-p-2 \\
j=0}}^{n-2}\left(z_{1}+\tau / \eta\right)^{j} z_{1}{ }^{n-p-2-\jmath}}{z_{1}{ }^{n-p-1}\left(z_{1}+\tau / \eta\right)^{n-p-1}}+O\left(\frac{1}{z_{1}^{n-p+1}}\right) .
\end{aligned}
$$

Assume $z_{1}=z_{0}+k \tau$ is a zero of $G(z)$. Then $L\left(z_{1}\right)$ is also a zero of $G(z)$ satisfying

$$
\left|L\left(z_{1}\right)-z_{1}\right|=\frac{\left|a_{p}\right|}{n}\left|1-\eta^{p}\right|\left|1-\eta^{n-1}\right| \frac{n-p-1}{|\tau|^{n-p-1}} \frac{1}{k^{n-p}}+O\left(\frac{1}{k^{n-p+1}}\right) .
$$

Hence $L\left(z_{1}\right)$ is a zero of $G(z)$ being very close to $z_{1}$. Hence around $z_{0}$ there is an infinite number of zeros of $G$. In order to prove this we make $L\left(z_{0}+k^{p} \tau\right), p=1,2, \cdots$ and reduce them around $z_{0}$ by subtracting $k^{p} \tau$. Then they are different and are close to $z_{0}$. This is a contradiction. Hence $a_{p}$ must be zero and hence

$$
h(z)=z^{n}+a_{0} .
$$

We next prove $n=1,2,3,4,6$. Let $z$ be a zero of $G$. Then $\{z+k \tau\}, k=0, \pm 1$. $\cdots$ is a set of zeros of $G(z)$. Further $\phi\left(\phi^{-1}(z)+\ell \tau\right)=z+\ell \eta \tau$ and $\phi^{2}\left(\phi^{-2}(z)+m \tau\right)=z$ $+\ell \eta^{2} \tau$ are also sets of zeros of $G(z)$. Therefore there are three vectors $\tau, \eta \tau, \eta^{2} \tau$ such that $z+p \tau+q \eta \tau+r \eta^{2} \tau$ indicates a set of zeros of $G$ for any triple of integers $p, q, r$. If $n \neq 1,2,3,4,6$, then Lemma 3 gives a cluster point of zeros of $G$, which is a contradiction. Thus we have $n=1,2,3,4,6$.

Next we prove that if $n=3,4$ or 6 then

$$
\text { ord } N(r, 0, G)=2 \text {. }
$$

In order to do it we pick up a zero $z$ of $G$. Then we have zeros of $G$ by $z+k_{0} \tau$ with integral coefficient $k_{0}$. Further $\phi(z)=\eta z$ is a zero of $G$ and hence $\left\{\eta z+k_{1} \tau\right\}$ is a set of zeros of $G$. Then returning back to $z$ by $\phi^{-1}(z)$ we have a set $\left\{z+\eta^{-1} k_{1} \tau\right\}$ of zeros of $G$. Repeat this process for $\eta^{p}, p=1,2, \cdots, n-1$. Then we have a set of zeros of $G$, whose form is $\left\{z+\eta^{-p} k_{p} \tau\right\}$. This set with $p=0,1, \cdots, n-1 ; k_{p}=0$, $\pm 1, \pm 2, \cdots$ is called the zero point lattice $\mathcal{L}(z)$ attached to $z$. We construct lattices for all zeros of $G$. $\mathcal{L}\left(z_{m}\right)$ may coincide with $\mathcal{L}\left(z_{\ell}\right)$ for $m \neq \ell$. This occurs if and only if $z_{\ell} \in \mathcal{L}\left(z_{m}\right)$. If there are infinitely many different lattices, then in any fundamental parallelogram $P$ with vertices $a, a+\eta^{-1} \tau, a+\eta^{-1} \tau+\tau, a+\tau$ there appear infinitely many zeros of $G$. Of course we consider $P$ as a torus. This is a contradiction. Each $\mathcal{L}\left(z_{m}\right)$ has $A_{m} r^{2}$ points in $|z| \leqq r$ with a positive constant $A_{m}$ if $r \geqq r_{0}$. Thus $N(r, 0, G) \sim A r^{2}$ with a positive constant $A$. Therefore ord $N(r, 0, G)=2$.

We prove ord $N(r, 0, G) \equiv \lambda$. ord $G \geqq \lambda$ is trivial. Assume that ord $G>\lambda$. Then by $\operatorname{ord} G<\infty \quad G(z)$ has the form

$$
B z^{v} e^{H(z)} \Pi E\left(z, a_{m}\right),
$$

where $E\left(z, a_{m}\right)$ is the Weierstrass prime factor 


$$
\left(1-\frac{z}{a_{m}}\right) \exp \left\{\frac{z}{a_{m}}+\frac{1}{2}\left(\frac{z}{a_{m}}\right)^{2}+\cdots+\frac{1}{q}\left(\frac{z}{a_{m}}\right)^{q}\right\}, \quad q=[\lambda]
$$

and $\Pi$ is taken over all zeros of $G$ excepting $a_{m}=0, \nu=0$ or 1 . Since ord $\Pi$ $=\operatorname{ord} N(r, 0, G)=\lambda$,

$$
\operatorname{deg} H \geqq[\lambda]+1>\lambda \text {. }
$$

We put $H(z)=a_{\mu} z^{\mu}+a_{\mu-1} z^{\mu-1}+\cdots+a_{1} z, a_{\mu} \neq 0, \mu \geqq[\lambda]+1$. Then the asymptotic behavior of $e^{H(z)}$ around $z=\infty$ is well known. It has

$$
e^{\Re a_{\mu \tau}{ }^{\mu} e i 2^{\mu_{\pi}(1+O(1 / r))} \text {. }}
$$

Thus on putting $a_{\mu}=\left|a_{\mu}\right| e^{i \theta_{\mu}}$

$$
\cos \left(\theta_{\mu}+2 \mu \pi\right)
$$

controlls the asymptotic behavior of $e^{H(z)}$. If $\cos \left(\theta_{\mu}+2 \mu \pi\right)>0$, then $e^{H(z)} \rightarrow \infty$ as

$$
\left|e^{H(z)}\right| \sim e^{|\alpha| r \cos \left(\theta_{\mu}+2 \mu \pi\right)}, \quad r \rightarrow \infty .
$$

If it is negative, then $e^{H(z)} \rightarrow 0$ as

$$
\left|e^{H(z)}\right| \sim e^{\left|a_{\mu}\right| r^{\mu} \cos \left(\theta_{\mu}+2 \mu \pi\right)}, \quad r \rightarrow \infty .
$$

$G$ is uniformly bounded for the $\tau$-direction, that is, $G$ is bounded along any curve $z=z_{0}+t \tau, t>0, t \rightarrow \infty$ and $\left|z_{0}\right| \leqq M$. On the other hand $|\Pi| \leqq e^{r^{2+\varepsilon}}, \varepsilon>0$ for $r \geqq r_{0}$. Further we can use the the following estimation [1, p. 73]: For every $\zeta>0, \xi>0$ there is a $K(\rho, \zeta, \xi)$ such that

$$
\log \left|\Pi\left(r e^{i \theta}\right)\right|>-K(\rho, \zeta, \xi) A V(r)
$$

for $\zeta R \leqq r \leqq R$, except perhaps in circles the sum of whose radii is at most $\xi R$, provided that $R>R_{0}(\zeta, \xi)$. Here $A$ is a constant and $V(r)=r^{p(r)}$ with the Lindelöf proximate order $\rho(r)$ of $\Pi$. Of course $\rho(r)<\lambda+\varepsilon$ for every $\varepsilon>0$ if $r \geqq r_{0}$. Hence in any sector in which $e^{H} \rightarrow \infty \quad G$ is not bounded. Further in any sector in which $e^{H} \rightarrow 0 \quad G$ tends to zero as $z \rightarrow \infty$. But $G$ does not tend to zero for the $\tau$-direction. Thus the $\tau$-direction should be a direction defined by $\cos \left(\theta_{\mu}+2 \mu \pi\right)=0$. Since $\mu \geqq[\lambda]$ +1 , there are $\eta^{\nu / 4} \tau, \eta^{\sigma / 4} \tau$-directions such that $\eta^{0 / 4} \neq \pm 1, \eta^{\alpha / 4} \neq \pm 1$ and $G \rightarrow 0$ uniformly as $z \rightarrow \infty$ along these two directions. These directions along which $G \rightarrow 0$ as $z \rightarrow \infty$ are equally spaced around $z=0$. Hence we may take them so that $\eta^{v / 4} \tau, \eta^{\sigma / 4} \tau$ lie in the opposite side with respect to the $\tau$-direction. Hence $G(z)$ is bounded in any half period strip by the periodicity of $G$ and so in the plane. This is a contradiction. Thus we have

$$
\operatorname{ord} G=\operatorname{ord} N(r, 0, G) \text {. }
$$

This completes the proof of Theorem 1 .

4. We shall show that theorem 1 is best possible. For $\nu=1$, it is trivial. For 
$\nu=2$, take $g=\cos \sqrt{w}, h=z^{2}, f=1$ and $G=\cos z$. For $\nu=3$ set

$$
\psi(z)=\prod_{-\infty}^{\infty}\left(1-\frac{\cos 2 \pi(z+n \omega)}{\cos 4 \pi n \omega}\right), \quad \omega=e^{2 \pi 2 / 3} .
$$

It is easy to verify the convergence of the infinite product in the right hand side. Let $M(R)$ be $\max _{|z|=R}|\phi(z)|$. Since $\phi(z)$ has period $1, M(R)$ is taken by $\phi$ at a point on the arc $|z|=R,|\Re z| \leqq 1 / 2$. Further $\phi$ is even. So we evaluate $|\psi(z)|$ for $z=x+i y,|x| \leqq 1 / 2, y>0$. We have

$$
|\psi(z)| \leqq|1-\cos 2 \pi z| \prod_{-\infty}^{\infty}\left(1+\frac{e^{n_{\pi} \sqrt{3}}+y+e^{-(n \pi \sqrt{3}+y)}}{e^{2 \pi|n| \sqrt{3}}-e^{-2 \pi|n| \sqrt{3}}}\right)
$$

and

$$
|1-\cos 2 \pi z| \leqq 1+\frac{e^{2 \pi y}+e^{-2 n y}}{2} \leqq e^{2 \pi y},
$$

where $\Pi^{\prime}$ indicates the product omitting $n=0$. Hence for large $y$

$$
|\psi(z)| \leqq e^{2 \pi y} \prod_{n=1}^{\infty}\left(1+\frac{e^{\pi(n \sqrt{3}+2 y)}+e^{-\pi(n \sqrt{3}+2 y)}}{e^{2 \pi n \sqrt{3}}-e^{-2 \pi n \sqrt{3}}}\right)^{2}
$$

and

$$
\begin{aligned}
\log |\psi(z)| & \leqq 2 \pi y+2\left(\sum_{y>2 \pi n \sqrt{3}}+\sum_{y \leqq 2 \pi n \sqrt{3}}\right) \log \left(1+\frac{e^{\pi(n \sqrt{3}+2 y)}+e^{-\pi(n \sqrt{3}+2 y)}}{e^{2 \pi n \sqrt{3}}-e^{-2 \pi n \sqrt{3}}}\right) \\
& \leqq \frac{y}{2 \pi \sqrt{3}}(2 \pi y+\log 2)+K y .
\end{aligned}
$$

Since $y \sim R$ for large $y$, we have ord $\phi \leqq 2$. On the other hand $\phi$ has double zeros at $z=3 n \omega$ and simple zeros at $z=(3 n+1) \omega,(3 n+2) \omega$. Considering the periodicity of $\phi$, we see that the convergence exponent of its zeros is equal to 2 . Hence ord $\phi=2$. We define $G$ as

$$
G=(\psi(z) \psi(z+\omega) \phi(z+2 \omega))^{1 / 4} .
$$

Let $\alpha_{\nu}$ be the images of the points $n+m \omega$ under $w=z^{3}$. Set

$$
g(w)=w \prod_{\nu=1}^{\infty}\left(1-\frac{w}{\alpha_{\nu}}\right)
$$

and

$$
\begin{aligned}
f(z)^{2} & =z^{3} \prod_{\nu=1}^{\infty}\left(1-\frac{z^{3}}{\alpha_{\nu}}\right) / G(z) \\
& =z^{2} e^{P(z)} .
\end{aligned}
$$

Thus a solution is constructed.

For $\nu=6$ we put 


$$
G(z)=\frac{(\psi(2 z) \phi(2 z+\omega) \phi(2 z+2 \omega))^{1 / 4}}{(\phi(z) \phi(z+\omega) \phi(z+2 \omega))^{1 / 4}}
$$

and replace the $\alpha_{\nu}$ by the images of zeros of $G$ under $w=z^{6}$. Set

$$
g(w)=w \prod_{v=1}^{\infty}\left(1-\frac{w}{\alpha_{\nu}}\right)
$$

and

$$
\begin{aligned}
f(z)^{2} & =z^{6} \prod_{\nu=1}^{\infty}\left(1-\frac{z^{6}}{\alpha_{\nu}}\right) / G(z) \\
& =e^{P(z)}
\end{aligned}
$$

Thus a solution is constructed.

For $\nu=4$ set

$$
\psi(z)=\prod_{-\infty}^{+\infty}\left(1+\frac{\cos 2 \pi(z+i(2 n-1) / 2)}{\cosh 4 \pi n}\right)
$$

and

$$
G(z)=(\phi(z) \psi(z+i) \phi(z+2 i))^{1 / 4} .
$$

By taking $\alpha_{\nu}$ in the expression of $g$ as the images of zeros of $G$ under $w=z^{4}$ and $h=z^{4}$, we get a solution.

5. Proof of Theorem 2. By Theorem 1

$$
\text { ord } G=\nu \text { ord } N(r, 0, g), \quad \nu=\left\{\begin{array}{lll}
1,2 & \text { if } & \text { ord } G \neq 2, \\
1,2,3,4,6 & \text { if } & \text { ord } G=2 .
\end{array}\right.
$$

Every periodic function is of order $\geqq 1$. Hence ord $g \geqq 1$. Further ord $g=\operatorname{ord} N(r, 0, g)$ by the proof of Theorem 1 , since $g$ has a zero and hence $\infty>\operatorname{ord} N(r, 0, g) \geqq 1$ by its periodicity. If ord $G=2$, then $\nu$ should be 1 or 2 . Hence in any case $\nu$ should be 1 or 2 . If $\nu=1$, then we have the desired result. Assume $\nu=2$, that is the degree of $h(z)$ is equal to 2 . Then we put

We have

$$
w \equiv h(z)=A(z-\alpha)^{2}+B, \quad A \neq 0 .
$$

$$
z=\alpha \pm \sqrt{\frac{w-B}{A}}
$$

Since $g(w)$ is periodic with period $s$ and $f(z)$ has only a finite number of zeros,

$$
z_{p}^{ \pm}=\alpha \pm \sqrt{\frac{w_{0}+p_{s}-B}{A}}, \quad p \geqq p_{0}
$$

are two zeros of $G(z)$ if $g\left(w_{0}\right)=0$. Hence 


$$
\begin{aligned}
& z_{p_{+1}}^{+}-z_{p}^{+}=\sqrt{\frac{s}{A}} \frac{1}{2 \sqrt{p}}+O\left(\frac{1}{p \sqrt{p}}\right), \\
& z_{p_{+1}}-z_{p}^{-}=-\sqrt{\frac{s}{A}} \frac{1}{2 \sqrt{p}}+O\left(\frac{1}{p \sqrt{p}}\right) .
\end{aligned}
$$

By the periodicity of $G(z)$ we have a cluster point of zeros of $G$, which is a contradiction. Thus we have the desired result.

6. Proof of Theorem 3. In order to prove Theorem 3 we again need the following fact: Let $\tau$ be the period of $G$. The $\pm \tau$ directions divide the plane into two sides. If $G(z)$ is bounded in a half period strip, then this is true in a half plane lying in one side which contains the half period strip.

Let $\nu$ be the degree of $P(z)$. We may assume that

$$
P(z)=z^{\nu}+p_{\nu-2} z^{\nu-2}+\cdots+p_{0}
$$

Consider the equation $P(z)=P\left(z_{0}+k \tau\right), k$ : integer $>0$. If $k$ is sufficiently large, then there are $\nu$ solutions $z_{\ell, k}, \ell=0,1, \cdots, \nu-1, z_{0, k}=z_{0}+k \tau$. Then

$$
z_{\ell, k}=\left(z_{0}+k \tau\right) e^{2 \pi i \ell / \nu}\left(1+O\left(\frac{1}{k^{2}}\right)\right) \text {. }
$$

Then

$$
\begin{aligned}
G\left(z_{\ell, k}\right) & =\frac{Q\left(z_{0, k}\right)}{Q\left(z_{\ell, k}\right)} G\left(z_{0, k}\right) \\
& =\frac{1+O\left(1 / k^{2}\right)}{e^{(2 \pi i \ell / \nu) q}} G\left(z_{0}\right), \quad q=\operatorname{deg} Q .
\end{aligned}
$$

This means that $G(z)$ is bounded uniformly for a direction

$$
\tau e^{2 \pi i \ell / \nu} \text {. }
$$

If $\nu \geqq 3$, then $\ell=1$ and $\ell=\nu-1$ give two directions which are not parallel to $\pm \tau$ and lie in the opposite side with respect to the $\tau$-direction. Hence $G(z)$ is bounded in both of half period strips. This is a contradiction.

7. Proof of Theorem 4. Evidently $f(z)$ has a finite number of zeros. If $f(z)$ is a polynomial, then theorem 2 gives the result. Hence $f^{2}(z)=Q(z) e^{H(z)}, \operatorname{deg} H$ <ord $G . \quad Q$ is a polynomial. Let $H(z)$ be

$$
h_{N} z^{N}+\cdots+h_{1} z,
$$

$q=\operatorname{deg} Q$. Then

$$
G\left(z_{\ell, k}\right)=\frac{1+O(1 / k)}{e^{(2 \pi i \ell) q / \nu}} \frac{e^{h} N_{0, k}^{N} k^{(1+O(1 / k))}}{e^{h N_{0, k} z^{2} e^{2 \pi i \ell N / \nu}}(1+O(1 / k))} G\left(z_{0}\right) .
$$

If $\nu \geqq 3$, then 


$$
\log G\left(z_{\ell, k}\right)=O\left(h_{N} z_{0, k}^{N}\left(1+O\left(\frac{1}{k}\right)\right)\right) .
$$

Thus by the periodicity of $G(z)$ in any direction

$$
\log G(z)=O\left(z^{N}\right) \quad \text { uniformly. }
$$

Hence

$$
m(z, G)=O\left(r^{N}\right) .
$$

Since $N<\operatorname{ord} G$, this implies a contradiction.

\section{REFERENCES}

[1] Cartwright, M. N., Integral functions. Cambridge tract (1962).

[2] Hiromi, G., ANd H. Mutō, On the existence of analytıc mappıngs, I. Kōdai Math. Sem. Rep. 19 (1967), 236-244.

[3] Niven, I., AND H. S. ZuCKerman, An introduction to the theory of numbers. John Wiley and Sons (1960).

[4] Ozawa M., On the existence of analytic mappings. Kōdaı Math. Sem. Rep. 17 (1965), 191-197.

[5] Ozawa, M., A remark on ultrahyperelliptic surfaces. Kōdai Math. Sem. Rep. 19 (1967), 381-383.

[6] RéNYI, A., ANd C. RÉNYI, Some remarks on perıodic entire functions. J. Analyse Math. 14 (1965), 303-310.

[7] v. D. WAERdEN, B. L., Modern algebra. Springer (1955).

Department of Mathematics,

ToKyo Institute of TeChNology. 